\title{
EFFECTS OF LETHALITY IN NAVAL COMBAT MODELS ${ }^{1}$
}

\author{
Michael J Armstrong \\ Sprott School of Business, Carleton University \\ Ottawa, ON, K1S 5B6
}

\begin{abstract}
In the context of both discrete time salvo models and continuous time Lanchester models we examine the effect on naval combat of lethality: that is, the relative balance between the offensive and defensive attributes of the units involved. We define three distinct levels of lethality and describe the distinguishing features of combat for each level. We discuss the implications of these characteristics for naval decision-makers; in particular, we show that the usefulness of the intuitive concept "more is better" varies greatly depending on the lethality level.
\end{abstract}

\section{INTRODUCTION}

One of the best-known mathematical models of combat is the Lanchester or Chase-Lanchester-Osipov (CLO) model, which has been used since 1902 to describe naval gun combat (see appendix $\mathrm{C}$ of [3]). This continuous time model uses differential equations to describe the evolution of a gunnery battle in which opposing units are continuously firing shells at each other. Because it is fairly simple and well understood by the combat modeling community, the basic CLO model has been widely studied (see e.g. [8]) and serves as the basis for other more advanced models (see e.g. [9]).

The basic CLO model unfortunately also has a number of weaknesses, particularly with respect to the modeling of land warfare (see [1] for a critique on this issue). With respect to naval warfare, the CLO models designed to represent historical gunnery combat are less suitable for describing battles between modern missile-bearing warships, where offensive missile fire tends to occur in discrete pulses or salvos, rather than continuously. To rectify this, Hughes [3] proposed a new discrete time salvo model in which offensive missile fire can be countered by a variety of defensive measures. The interaction between the offensive and defensive components in this model is intuitively appealing; however, this same interaction means that model analysis is more complex than that for CLO gunnery models. As Hughes stated [3, p 270], "Exploratory calculations indicate that results are very parameter dependent, that is, case specific. In fact, case-by-case results vary so widely as to strongly suggest that detailed simulation will be vacuous until a clearer pattern of both the analytical and operational behavior of the various attributes is grasped first."

\footnotetext{
1 This is the peer reviewed version of the following article: Armstrong MJ, 2004, "Effects of lethality on naval combat models", Naval Research Logistics 51, 28-43, which has been published in final form at http://onlinelibrary.wiley.com/doi/10.1002/nav.10102/abstract. This article may be used for non-commercial purposes in accordance with Wiley Terms and Conditions for Self-Archiving.
} 
The objective of our research herein is to provide a better understanding of modern naval combat as represented by the discrete time salvo model, and to do so in a way that is inclusive of the more established continuous time CLO model as well. We do this by proposing a classification scheme based upon what we call the lethality of the combat situation: that is, the relative balance between the offensive and defensive capabilities of the opposing unit types. Our definitions and descriptions are framed in such a way as to apply to both the salvo model and the CLO model, and to include (in a simple form) a variety of weaponry, such as missiles, shells, or torpedoes.

We begin in the next section by introducing our modeling framework, and show how it relates to the basic versions of both the continuous time CLO model and the discrete time salvo model. Using this framework we then define three distinct levels of combat lethality. We describe the characteristics of combat at each level of lethality, including the calculation of losses and the relative difficulty of achieving mission objectives. We find that while moderate lethality combat behaves in a fairly straightforward manner, both low lethality combat and high lethality combat can exhibit less intuitive behavior. In particular, the simple philosophy of "more is better" that works well with moderate lethality gun combat is shown to be problematic when low or high lethality combat occurs. We provide some numerical examples to illustrate our results, and conclude with a discussion of the implications of our work for naval decision makers. The mathematical derivations underlying our results are provided in an appendix.

\section{MODEL FRAMEWORK}

\section{Notation}

Consider two naval task forces A and B that meet and engage in battle. As in other studies (e.g. [3], [8]) for simplicity we assume that each side has a homogenous force composed of a single unit type; thus all the units on side A share the same characteristics, and all the units on side B likewise have their own common parameter values (see e.g. [6] for one way to handle heterogeneous forces). The two forces exchange simultaneous fire until all units on one side are knocked out of battle. We ignore any considerations of ammunition supplies, detection \& evasion, etc; and since naval combat is our primary interest, we also ignore suppression and other psychological effects of battle (see [2] or [4] for discussions of suppression in the context of land battles).

Our framework for modeling this battle is built largely upon the basic salvo model in section III of [3] with some minor notation changes; the reader may wish to refer to that paper for a more thorough discussion of its terminology and underlying assumptions. Let parameter $A$ represent the force strength or number of units on side A as of time $t$, where $t=0$ represents the start of the battle. By units we mostly mean surface vessels, but this may be extended to include other weapon platforms such as helicopters or submarines in those limited cases where their distinctive maneuver and concealment capabilities are not relevant. We can use $A_{t}$ to indicate when we are specifically discussing a discrete time model, or $A(t)$ when specifying a continuous time model. Each 
unit of A has an offensive power (or fighting power) rating $\alpha$, which is the number of well-aimed rounds fired by each combatant per unit time at the opposing force; a round may be a shell, torpedo, or surface-to-surface missile (SSM) according to the situation. Each of A's units also has a staying power value $w$ that represents the number of hits needed to achieve a firepower kill on it (i.e., to put it out of action, though not necessarily sink it). Finally each unit has a defensive power value $y$, which is the number of incoming well-aimed rounds prevented from hitting by active defenses per defending combatant per unit time. This is primarily composed of counter-fire that intercepts incoming rounds via e.g. surface-to-air missiles (SAMs). The defensive power value will typically be zero if the incoming fire is in the form of shells or torpedoes, and positive if the incoming fire is composed of SSMs. The units on side B are similarly represented by parameters $B, \beta, x$, and $z$, respectively; see Table 1 for a summary of model parameters.

Table 1. Model Parameters

\begin{tabular}{|lll|}
\hline Side A & Side B & Description \\
\hline$A$ & $B$ & Quantity of units at time $t$ \\
\hline$\alpha$ & $\beta$ & Offensive power per unit \\
\hline$w$ & $x$ & Staying power per unit \\
\hline$y$ & $z$ & Defensive power per unit \\
\hline
\end{tabular}

\section{Discrete Time Equations}

Since combat is inherently a discrete phenomena ([1]) consisting of ships, shells, etc, it is most natural to model it using a discrete time model, as was done in [3] and [6]. In this context, we assume that each force fires a simultaneous salvo of rounds at the other force in each time interval, so that the evolution of the combat is a series of salvos or pulses of firepower. The change in force strength for each side during time interval $t$ can then be expressed using the difference equations from [3] (with notation adapted appropriately):

$$
\Delta A_{t}=-\left(\beta B_{t}-y A_{t}\right) / w, \quad 0 \leq-\Delta A_{t} \leq A_{t} ; \quad \Delta B_{t}=-\left(\alpha A_{t}-z B_{t}\right) / x, \quad 0 \leq-\Delta B_{t} \leq B_{t} .
$$

The number of units lost by each side in time interval $t$ is thus proportional to the number of incoming rounds that are not intercepted by friendly defensive power. If there is no defensive power (as with gun and torpedo combat), then $y=z=0$ and all offensive fire in each time interval causes damage; this was the case considered in the analysis of historical naval battles in [5] and [6].

To find a battle's final result, i.e., the number of survivors on each side, we can use an iterative procedure to update each side's strength until one of them drops to zero.

$$
\begin{array}{ll}
A_{t+1}=A_{t}-\left(\beta B_{t}-y A_{t}\right) / w=(1+y / w) A_{t}-(\beta / w) B_{t}, & 0 \leq A_{t+1} \leq A_{t} \\
B_{t+1}=B_{t}-\left(\alpha A_{t}-z B_{t}\right) / x=(1+z / x) B_{t}-(\alpha / x) A_{t}, & 0 \leq B_{t+1} \leq B_{t}
\end{array}
$$

Later we show situations where these iterations can be replaced by a single calculation. 


\section{Continuous Time Equations}

When the amount of damage caused by each round is small relative to the staying power of the units involved (as in most gun combat) it can be convenient to use a continuous time approximation of combat. By taking the limit as a time interval goes to zero, $\Delta t \rightarrow 0$, we get a system of linear differential equations; this implies that we are treating both offensive and defensive power as continuous streams rather than as discrete events. In this case the system of difference equations from the previous section becomes the following system of differential equations.

$$
\frac{d}{d t} A(t)=-[\beta B(t)-y A(t)] / w, \quad \frac{d}{d t} A(t) \leq 0, \frac{d}{d t} B(t)=-[\alpha A(t)-z B(t)] / x, \quad \frac{d}{d t} B(t) \leq 0
$$

If there is no defensive fire (as with torpedoes or guns), then $y=z=0$ and these equations simplify to standard CLO "square law" equations, whose solution is well known (see e.g. [8]). The solution of our somewhat more general system here also exists but is slightly more complex, so we present it in the appendix.

\section{LETHALITY CATEGORIES}

\section{Definition}

One of the main points of [3] was that modern naval forces are often capable of inflicting such large amounts of damage with just a single salvo of missiles that the nature of naval combat has been significantly altered; combat has in some sense become more lethal. This suggests that combat lethality is a feature worthy of further study. In order to make the concept of lethality useful however, we need to define it more precisely. Our discussion will be cast in terms of the relative force strength $B / A$; that is, the ratio of the number of units in force $B$ to that in force $A$. One reason for choosing this formulation is that operational decision makers can generally exert considerable control over the quantity of units committed to a particular battle. In contrast, they have little short-term control over the individual unit qualities, as these are largely fixed in advance of a campaign by ship design and training decisions (these factors were emphasized in [3]). We may think of our analysis from the following point of view: the commander of side $\mathrm{B}$ knows the strength $A$ of the enemy force, and is now considering the ramifications of choosing the size of force $B$ to send to oppose it.

We begin our definition effort by supposing that each side is unable to penetrate the other's defenses; that is, $A \alpha-B z \leq 0$ and $B \beta-A y \leq 0$. These conditions imply $\alpha / z \leq$ $B / A$ and $B / A \leq y / \beta$, respectively. If $\alpha / z \leq y / \beta$ then there is a set of $B / A$ values (possibly just a single point) where neither side can penetrate the opponent's defenses. Conversely, if $\alpha / z>y / \beta$ then for any given $B / A$ value at least one side is able to inflict damage on their opponent. 
Next let us suppose instead that each side is able to eliminate the other side with just one salvo; that is, $(A \alpha-B z) / x \geq B$ and $(B \beta-A y) / w \geq A$. These conditions imply that $\alpha /(x+z) \geq B / A$ and $B / A \geq(w+y) / \beta$. If $(w+y) / \beta \leq \alpha /(x+z)$ then there is a set of $B / A$ values (possibly just a single point) where both sides are simultaneously eliminated in one salvo. Conversely, if $(w+y) / \beta>\alpha /(x+z)$ then for any given $B / A$ value at most one side is able to eliminate their opponent in a single salvo.

With these two pairs of ratios we can now classify the lethality of a given combat situation into one of three categories:

(a) Low lethality combat has $\alpha / z \leq y / \beta$;

(b) Moderate lethality combat has $\alpha / z>y / \beta$ but $\alpha /(z+x)<(y+w) / \beta$; and,

(c) High lethality combat has $\alpha /(z+x) \geq(y+w) / \beta$.

In the following sub-sections we describe in detail these three different levels of lethality.

Notice that these definitions compare the attributes of a specific pairing or matchup of two opposing unit types, one unit type on each side. This classification holds for any quantities of these two given unit types that might be committed to battle, but it must be re-calculated if a different type of unit is substituted on either or both sides. Our definition therefore accepts that combat is inherently a force-on-force process ([1] \& [3]), where the effective worth of a combat unit is not an absolute to be evaluated in isolation, but rather must be determined in relation to a specific opponent. It is also worth noting that we are using the term lethality here in a relative sense: a battle that is "low" in lethality may still have extensive losses on one side, while a battle that is "high" in lethality may nonetheless have survivors on one side.

\section{Moderate Lethality Combat}

We define a combat situation to have moderate lethality if $\alpha / z>y / \beta$ but $\alpha /(z+x)<$ $(y+w) / \beta$. The first pair of ratios checks to ensure that what we might call side A's "relative offensive power" is at least as great its "relative defensive power"; the second pair of ratios checks that what we could call its "relative adjusted offensive power" is less than its "relative adjusted defensive power". These interpretations are easier to see in the special case where both sides have the same ship type (i.e. with matching parameters $\alpha=\beta, w=x$, and $y=z$ ). In that case the definition simplifies to $\alpha>y$ but $\alpha<(y+w)$; that is, each ship has sufficient offensive power to penetrate one target's active defenses and inflict some damage, but not enough to achieve an outright firepower kill.

We consider the moderate lethality situation first because it is likely the one that is most familiar; it covers almost all naval gunnery battles as modeled by e.g. CLO differential equations. With current technology, gun battles have negligible active defenses $(y=z=0)$ and thus $\alpha / z \rightarrow \infty$ is always greater than $y / \beta \rightarrow 0$. At the same time, the amount of damage per salvo is typically quite small compared to total staying power, so that $\alpha /(z+x)=\alpha / x$ is generally much less than $(y+w) / \beta=w / \beta$. Missile combat would fit here when each unit has roughly equal SSM and SAM capacities per salvo. Torpedo 
combat would be classified here only if technology were such that a vessel requires multiple torpedo hits to knock it out (as with perhaps capital ships in World War II).

In this situation it is useful to define a force strength parity point $P$ that indicates when two forces are evenly matched; i.e., if $B / A=P$ then the two forces will gradually wipe each other out over the course of the battle (i.e., they eventually achieve mutual destruction). This is equivalent to having a fractional exchange ratio (described in [3]) exactly equal to one. The formula for the parity point (derived in the appendix) is

$$
P \equiv \sqrt{\frac{\alpha}{\beta} \frac{w}{x}(1+s)}, \quad \text { where } s \equiv \frac{(w z-x y)^{2}-(w z-x y)\left[(w z-x y)^{2}+4 \alpha \beta w x\right]^{1 / 2}}{2 \alpha \beta w x} .
$$

The expression for $s$ represents the higher-order "interaction" effects of offensive, defensive, and staying power parameters for both sides. The formula for $P$ simplifies to yield a familiar result in two special cases:

(a) When $y=z=0$, i.e. defensive power is negligible, as in gun combat; or, (b) When $y / z=w / x$, i.e. the ratio of defensive power values equals the ratio of staying power values. This holds when the two unit types make similar use of defensive power and staying power in their defenses (as opposed to A being stronger on one factor while $\mathrm{B}$ is stronger on the other).

In either of these situations we get $s=0$ and the formula for parity simplifies to

$$
P=\sqrt{\frac{\alpha}{\beta} \frac{w}{x}}, \text { and thus parity implies that } B^{2} \frac{\beta}{w}=A^{2} \frac{\alpha}{x},
$$

which is simply the "square law" property we are accustomed to seeing in CLO models for gun combat. In either form the interpretation of $P$ is the same: if the ratio of force strengths is greater than parity $(B / A>P)$, then only $\mathrm{B}$ will have survivors left at the end of combat; if the ratio is less $(B / A<P)$, then only A will have survivors.

Now let us consider the implications of moderate lethality on the results of combat, in particular with respect to side B's decision of how many units to send into battle against a given enemy force. By comparing the force strength ratio $B / A$ to our defining ratios and the parity point, we can divide the spectrum of possible strength ratios into five regions according to the final results of the battle (see Figure 1):

(a) If $\mathrm{B}$ is badly inferior in relative strength, so that $B / A \leq y / \beta$, then its forces will be completely eliminated without causing any damage to side A units;

(b) If $\mathrm{B}$ is somewhat inferior, so that $y / \beta<B / A<P$, then its forces will be completely eliminated but at least some damage will be inflicted upon side $\mathrm{A}$;

(c) If $\mathrm{B}$ is exactly at strength parity with $\mathrm{A}$, so $B / A=P$, then both sides will be completely eliminated (after two or more salvos, in the discrete time case); 
(d) If $\mathrm{B}$ is somewhat superior in relative strength, so $P<B / A<\alpha / z$, then it will sustain some damage but will completely eliminate side $\mathrm{A}$; and,

(e) If $\mathrm{B}$ is greatly superior, so $\alpha / z<B / A$, then it will completely eliminate side $\mathrm{A}$ without sustaining any damage itself.

Figure 1. Losses to Each Side in Moderate Lethality Combat

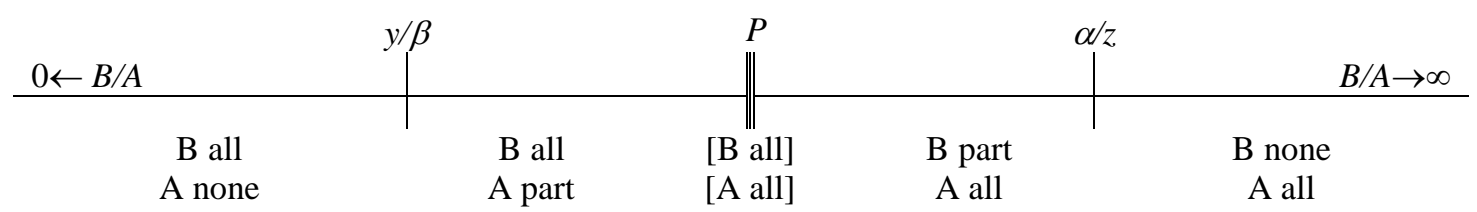

Where there is no defensive power (as with gun or torpedo combat) the first and last regions listed above do not exist, as $\alpha / z \rightarrow \infty$ and $y / \beta \rightarrow 0$; therefore the stronger force will always suffer at least some damage before the weaker force is eliminated, and it is always advantageous to have more ships rather than fewer ships. This "more is better" characteristic is one we are accustomed to seeing in CLO models and is a good rule of thumb to follow in general for moderate lethality combat, as the progression from one region to the next is rather gradual. For example, within the second region above as B adds more ships to its force, it will gradually cause more and more damage to A, up until parity is achieved; if B continues to add ships beyond this point, then its own losses will gradually decline. Achieving surprise in this situation (in the form of being able to fire one unanswered salvo at the start of battle) would of course be advantageous but depending on force strengths it may or may not be critical to the final result.

Commonly in CLO models we refer to the force with survivors as the winner, but it can be useful to distinguish other measures of "winning". In particular let us consider four prototypical mission objectives that a side might wish to achieve:

(a) Sea control. At the end of battle, there are at least some friendly survivors (to continue patrolling the area) but no enemy survivors (which could otherwise raid friendly shipping lanes);

(b) Sea denial. At the end of battle, there are no enemy survivors;

(c) Screening, as in a task force that is defending a convoy from attack while it transits the area. At the end of battle, there are at least some friendly survivors (to continue the screen);

(d) Attrition. The friendly task force trades casualties with the enemy on a "favorable" basis. By "favorable" we mean a ratio better than that obtained at force parity.

In the case of moderate lethality combat, these four mission types seem trivial to distinguish because they have almost identical requirements for success. Sea control, sea denial, screening, and favorable attrition are all achieved by $\mathrm{B}$ if the strength ratio exceeds parity $(B / A>P)$, and all fail if the strength ratio is less than parity $(B / A<P)$. In the unlikely event that exact parity is met, sea denial succeeds while sea control and screening fail. 
We can determine whether a force will have survivors or succeed at each of the above objectives simply by comparing the initial strength ratio $B / A$ to the range limits shown above. If we want to calculate the number of survivors, we either need to iterate the discrete time difference equations shown previously, or (if the amount of damage per round is small relative to staying power) use the corresponding continuous time differential equations.

\section{High Lethality Combat}

We define a combat situation to have high lethality if $\alpha /(z+x) \geq(y+w) / \beta$; i.e., if "relative adjusted offensive power" is greater than "relative adjusted defensive power" (this implies that $\alpha / z>y / \beta$ also). In the special case where both sides have the same unit type (i.e., with identical parameters), then this definition simplifies to $\alpha \geq(y+w)$; that is, each ship has sufficient offensive power to overpower a target's active defenses and achieve a firepower kill on it with a single salvo.

The high lethality situation was the one emphasized in the numerical examples of [3]. Missile combat fits here when the units have greater offensive (SSM) capacities than defensive (SAM) capacities per salvo, as with perhaps ships like the Arleigh Burke DDG class or with missile attack boats. Torpedo combat would typically fit here, as would some of the historical carrier battles analyzed in [5]. Gun battles generally would not be included, except perhaps in some very rare circumstances (perhaps e.g. an engagement at point blank range between large-gunned but lightly armored battle cruisers, where a single broadside could conceivably cripple an opposing vessel).

By comparing the relative force strength ratio $B / A$ to our defining ratios we can divide the spectrum of possible combat results into five regions somewhat similar to those of the moderate lethality case (see Figure 2):

(a) If $B / A \leq y / \beta$, B will be completely eliminated in one salvo without causing any damage to A's units;

(b) If $y / \beta<B / A<(y+w) / \beta$, B will be completely eliminated in one salvo but A will suffer some losses;

(c) If $(y+w) / \beta \leq B / A \leq \alpha /(z+x)$, then both sides will be completely eliminated in one salvo;

(d) If $\alpha /(z+x)<B / A<\alpha / z$, then B will sustain some damage but will completely eliminate side A in one salvo; and,

(e) If $\alpha / z \leq B / A$, then $\mathrm{B}$ will completely eliminate side $\mathrm{A}$ in one salvo without sustaining any damage itself.

Figure 2. Losses to Each Side in High Lethality Combat

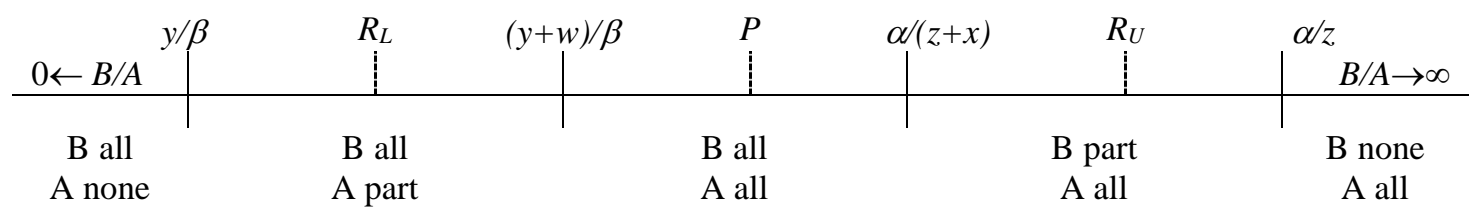


With respect to these regions, the largest difference between moderate and high lethality combat is the expansion of the mutual destruction result from a single point at $P$ (where destruction requires two or more salvos in the discrete time model) to a wider region of "effective" parity (where elimination requires only a single salvo). The width of this region grows as offensive power increases; in the limit as $\alpha \rightarrow \infty$ and $\beta \rightarrow \infty$ (e.g. with nuclear warheads) a single vessel is in principle sufficient to wipe out any enemy force it encounters, in exchange for its own loss. Note that the location of the parity point $P$ is not of direct significance here.

Another difference here is that no matter how many units are present the battle will be finished after only a single salvo, so we can calculate the number of survivors (if any) with just one iteration of the discrete time equations. It would be clearly inappropriate in this case to use the continuous time equations, as the offensive power is very high relative to the defense. Surprise in this situation could be crucial, as a single unanswered salvo could end the battle in favor of even a much inferior force.

We can determine which forces will succeed in completing their missions simply by considering the initial strength ratio $B / A$, but the strength ratio required for success will vary with the mission objective. The potentially large range of ratios that result in mutual destruction means that sea control and screening become harder to achieve (since they require some friendly forces to survive) while sea denial becomes easier.

The fourth mission objective, favorable attrition, is more complex in a high lethality situation. Recall that we say relative losses "favor" B if the ratio of the quantity of units lost by B to that of A is less than what occurs at force strength parity; in a sense we are using the parity point as a measure of the relative combat worth of A's units compared to B's. For example, if the parity ratio is 2 , then at force parity side B loses twice as many units as side A, and we might say that each A unit has twice the combat worth of each B unit for this particular pairing of unit types. Any strength ratio that results in $\mathrm{B}$ losing less than twice as many units as $\mathrm{A}$ is then said to favor $\mathrm{B}$, while a situation that results in $\mathrm{B}$ losing more than twice as many units as $\mathrm{A}$ is said to favor $\mathrm{A}$.

Under high lethality there are two other ratios of force strengths $R_{L}$ and $R_{U}$ that give the same trade-off of losses as would be obtained at the parity point $P$. These are found to be

$$
R_{L}=\frac{y}{\beta+w / P} \quad \text { and } \quad R_{U}=\frac{\alpha-x P}{z}
$$

where $R_{L} \leq P \leq R_{U}$ (see the appendix for their derivation). These three points allow us to indicate when losses will favor one side or the other. The strength ratios which favor A are $0<B / A<R_{L}$ and $P<B / A<R_{U}$, while losses favorable to $\mathrm{B}$ occur in the regions $R_{L}<$ $B / A<P$ and $R_{U}<B / A<\infty$ (see Figure 3). For each side there is a range where significant superiority yields a favorable exchange of losses, but also a second region in which slight inferiority also allows a favorable loss trade-off. This is the situation that Hughes 
illustrated in the numerical examples in [3]: under high lethality conditions a weaker force can conduct a successful "suicide attack" on a stronger opponent, as the superiority of the stronger side is effectively wasted. Note that where there is no defensive fire (e.g. in torpedo combat) these ratios diverge to $R_{L} \rightarrow 0$ and $R_{U} \rightarrow \infty$; thus in this situation the inferior force always benefits from an advantageous exchange of losses.

Figure 3. Relative Losses in High Lethality Combat

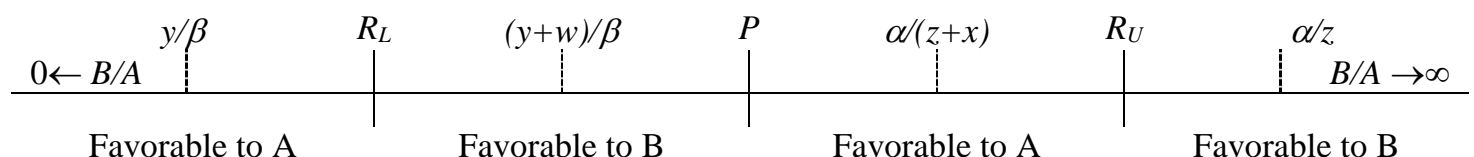

\section{Low Lethality Combat}

We define combat as having low lethality if $\alpha / z \leq y / \beta$ (which implies that $\alpha /(z+x)$ $<(y+w) / \beta$ as well $)$; i.e., the "relative offensive power" of side A's units is less than their "relative defensive power". If both sides have identical units, then this definition simplifies to $\alpha \leq y$; that is, each unit lacks sufficient offensive power to penetrate another unit's active defenses. This category would describe missile combat between units that have weaker offensive (SSM) capacities than defensive (SAM) capacities per salvo, as with perhaps air defense ships like the Ticonderoga CG class. Neither torpedo combat nor gun combat would fall into this category with current technology, since they both have zero defensive power values.

The relation between the initial strength ratio $B / A$ and the final combat result is quite different in this case from either moderate or high lethality combat; see Figure 4. There are only three regions in this case:

(a) If $B / A \leq \alpha / z$, then $\mathrm{B}$ will be completely eliminated without causing any damage to $\mathrm{A}$;

(b) If $\alpha / z \leq B / A \leq y / \beta$, then a standoff results and neither side takes any losses;

(c) If $y / \beta<B / A$, then $\mathrm{B}$ will completely eliminate A without sustaining any damage itself.

Figure 4. Losses to Each Side in Low Lethality Combat

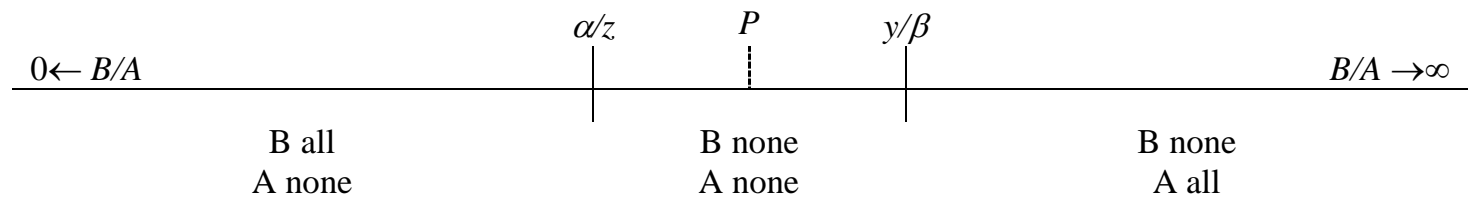

Because the results in each region vary dramatically for low lethality combat, the progression from one region to the next is very abrupt. B could add several more units to its force without making any difference at all to the outcome of the battle, if those additions left it in the same region; but the addition of even a single unit could make an enormous difference if it caused the force ratio to cross over one of the listed thresholds. Thus the principle of "more is better" is not strictly true here; more is only better if it is "enough more". 
We can determine whether a side will have survivors at battle's end by comparing the initial strength ratio $B / A$ to the range limits shown above. Calculation of the quantity of survivors is also trivial, as the initial strength ratio indicates which side (if any) is completely eliminated and which side(s) are unharmed.

With respect to mission completion, the different mission types again diverge somewhat with regards to the strength ratios required for success. The potentially large range of ratios that result in a loss-less standoff means that sea control and sea denial become harder to achieve (since they require the opponent's elimination) while screening missions become easier. The force ratio required for favorable attrition rates is higher and so harder to achieve (an inferior force never achieves favorable attrition); on the other hand, once such sufficient superiority is achieved the casualty exchange is ideal in that the enemy force is eliminated at no cost in friendly losses. Surprise in the sense of an unanswered salvo of missiles in this situation would not change the end result at all; on the other hand, achieving "complete" surprise in the sense of catching the opposing force with their defenses turned off would be particularly valuable since it could avoid a potential stalemate.

Where the forces are such that a loss-less standoff is the predicted result, in reality it is likely that something outside the model would occur to upset the uneasy missileversus-missile balance. For example, the situation would change if one side eventually ran out of missiles, received reinforcements sufficient to tip the balance, or closed the range for a follow-up battle with guns or torpedoes; in this latter case, the changeover to different weapons would require re-calculation of the ratios (e.g. a new value for the parity point $P$, etc) to model the new phase of the battle. Alternatively, a "lucky" missile could "leak" through the defenses to cause damage; the deterministic combat models we consider herein assume that defenses are perfect up to their capacity limit, whereas in reality there is always a chance they will miss (i.e., real combat is stochastic).

As an aside we note that some nations might prefer low lethality combat for political or social reasons, as it implies that sufficient force superiority not only achieves military objectives but also avoids any loss of friendly forces.

\section{NUMERICAL EXAMPLE}

In this section we illustrate our analytical results with a numerical example of a battle amongst missile-armed ships. For side A let staying power $w=4$ hits per ship, defensive power $y=8$ missile intercepts per salvo per ship, and offensive power $\alpha=4$ well-aimed missiles per salvo per ship. On side B we let $x=2, z=4$, and $\beta=2$. These figures give critical ratios $4 / 4<8 / 2$ and $4 /(4+2)<(8+4) / 2$, so they fit the description of low lethality. The parity ratio $P=2$ confirms that in this particular match-up each A ship is effectively twice as valuable as each B ship.

Suppose that A has a fixed task force of size $A=6$ ships. Figure 5 illustrates the results of battle by plotting the percentage of ships surviving on each side as the number 
of ships $B$ is varied from 4 to 28; diamonds represent A's survivors, while squares represent those of $B$. We see that $B$ eventually loses all of its ships for task forces of up to 5 ships; once B has a task force of 6 ships, it is able to shield itself from A's missiles and avoid any losses. To penetrate A's missile defenses and inflict losses however requires that $\mathrm{B}$ dramatically increase its task force up to at least 25 ships; smaller increases merely maintain the standoff, while increases beyond 25 provide no further benefit.

Figure 5. \% Force Surviving in Low Lethality Combat Example

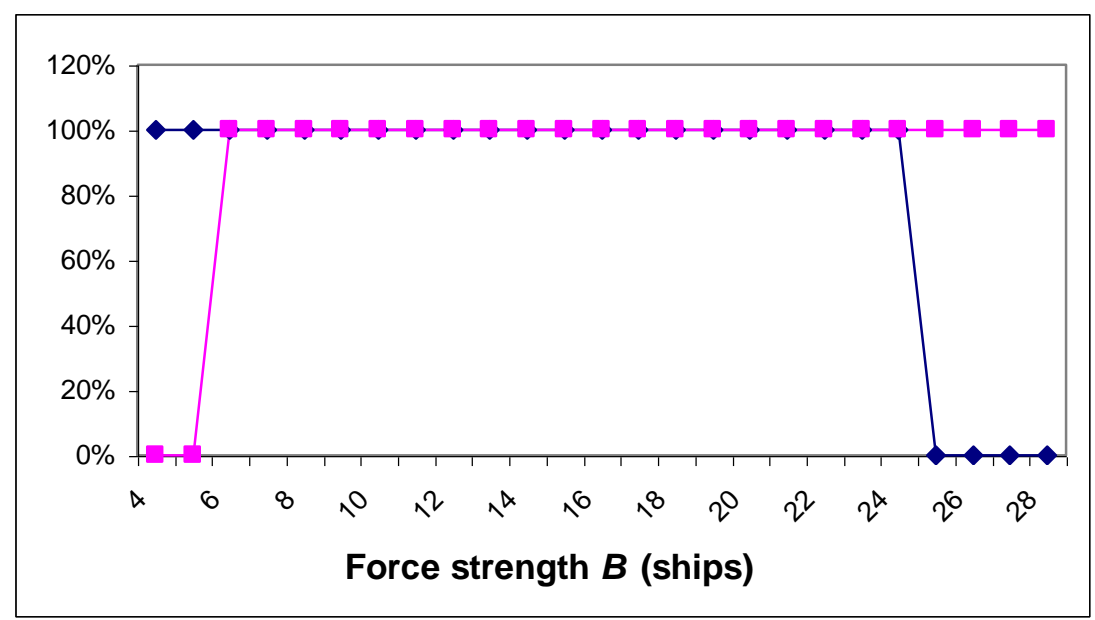

Figure 6. \% Force Surviving in Moderate Lethality Combat Example

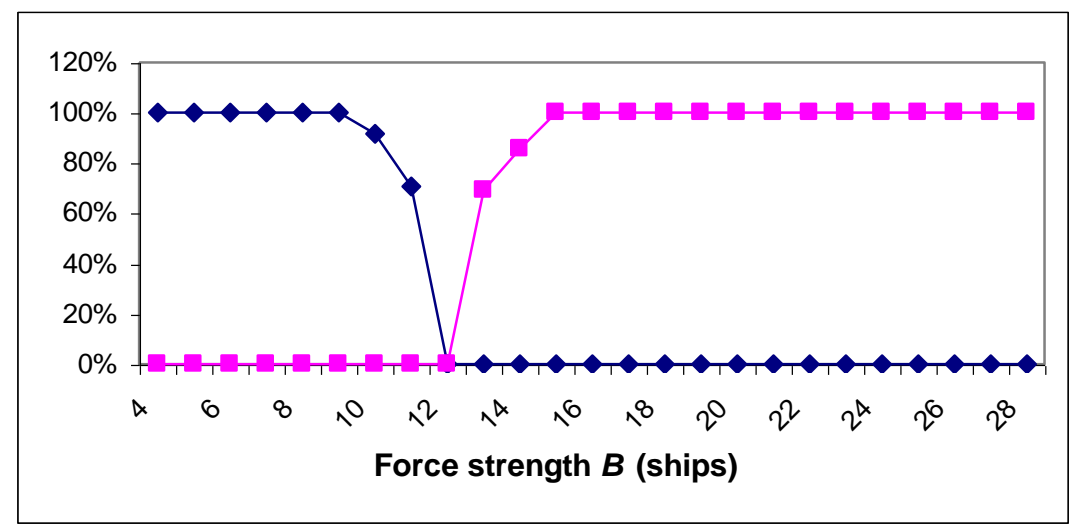

Next let us increase the offensive power ratings on both sides by setting missiles per salvo per ship at $\alpha=10$ and $\beta=5$. Then the defining ratios become $10 / 4>8 / 5$ and $10 /(4+2)<(8+4) / 5$, so the combat fits our moderate lethality definition; the parity ratio is still $P=2$. Figure 6 illustrates this case; survivors were calculated using several iterations of the discrete time equations for each data point. Initially an increase in $B$ only serves to lose more ships, but with the addition of the 10th ship B starts to inflict losses on side A. Further increases gradually shift the tide of battle: at 12 ships B has achieved parity $(12 / 6=2)$ and both sides are eliminated, while strength increases beyond that point serve to reduce B's own losses. 
Figure 7 illustrates the high lethality case, where we further increase missiles per salvo per ship to $\alpha=18$ and $\beta=9$. This gives defining ratios $18 / 4>8 / 9$ and $18 /(4+2)>$ $(8+4) / 9$. In this case as we increase B's strength it continues to lose all of its ships up until the 18th ship; only with the addition of the 19th ship does B start to reduce its losses. On the other hand, B begins to inflict losses on side A earlier than in the previous cases, and $\mathrm{A}$ is completely eliminated once $\mathrm{B}$ has 8 ships. When $\mathrm{B}$ has from 8 to 18 ships both sides are knocked out after a single salvo. With respect to the relative exchange of casualties, the parity point is again $P=2$ while the other key ratios are $R_{L}=1.143$ and $R_{U}$ $=3.5$. Thus B is "ahead of the game" with respect to attrition for task force sizes from 7 to 11 ships, and then again from 22 ships and upwards. Outside those ranges the relative ship losses are either neutral (at $B=12$ ships and again at $B=21$ ships) or else favor A.

Figure 7. \% Force Surviving in High Lethality Combat Example

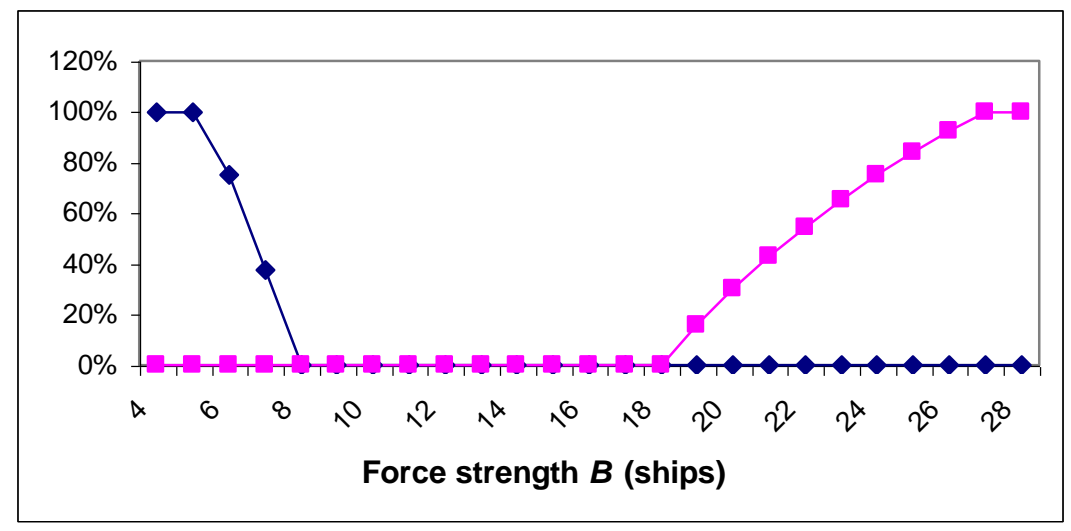

\section{DISCUSSION}

The three levels of combat lethality we have proposed in the preceding sections arise as offensive power gradually increases relative to defensive and staying power; as we look from Figures 4 to 1 to 2 , we see for example the ratio $\alpha / z$ gradually moving from left to right and crossing over the ratio $y / \beta$ as it gradually shifts from right to left. Interestingly however the three resulting lethality levels are not merely progressive variations of one another but rather are quite distinctive. Low lethality combat for example has a loss-less "stand-off" region at its center, and permits a slightly inferior force to survive without harm. Conversely, a low lethality situation could frustrate a navy's desire to achieve a decisive result against what is by most measures an inferior opponent. Moderate lethality combat has neither of these features; instead it has a single critical point $P$ at which both sides are eventually eliminated, and it denies an inferior force any measure of success. In contrast to both of these cases, high lethality combat has an extended range over which both forces are immediately eliminated, and somewhat perversely allows a slightly inferior force to accomplish some mission objectives.

We believe this categorization of lethality is of relevance not just for historical analysis ([5] describes several historical examples that illustrate the lethality effects 
defined herein), but also for contemporary naval decision-making. For example, where high lethality combat is the norm it is possible at least in principle for a navy to wage a victorious (albeit costly) campaign even though it is outnumbered and outclassed in every single battle it fights. Consider the following case: A has 21 ships available in theatre, each one having $\alpha=18, w=4$, and $y=8$; and B has 19 ships, each with $\beta=14, x=3$, and $z=6$. This is high lethality combat, and $P=1.31$ confirms that A's ships are superior to B's; thus at the theatre level $\mathrm{B}$ is outclassed $31 \%$ ship-for-ship and also outnumbered by $11 \%$, so we might reasonably expect $\mathrm{B}$ to lose the campaign.

Now suppose that by skill or luck side $B$ arranges a set of 3 battles, each with $A=7$ and $B=6$ (so all A ships are committed, but $1 \mathrm{~B}$ ship is held in reserve). In each battle $\mathrm{B}$ is outnumbered and outclassed, but because of the high lethality both sides are eliminated in each engagement. After the 3 battles are completed, A has no ships left in fighting condition but $\mathrm{B}$ still has 1 available to accomplish its missions and win the campaign. Note that this example is different from the classic case of a weaker side winning by achieving local superiority; here, the weaker navy has local inferiority in every battle and yet still wins overall simply by keeping a reserve out of harm's way.

Faced with this these results, a modeler or commander might well ask which factor is most important to victory (or equivalently, which parameter is most worth improving)? As noted in [3], the relative complexity of combat involving defensive power makes answering this question harder than in the original CLO context where the much-debated square law holds. Nonetheless, our three-level classification allows us to offer at least some advice for a naval planner:

(a) In low lethality combat, improving defensive power is important for avoiding defeat while improving offensive power is important for enabling victory (see the ratio locations on Figure 4). Force strength is important but problematic, as changes to it only influence the battle's outcome if they are "large enough". Staying power is irrelevant to the final result;

(b) In moderate lethality combat we saw earlier that the square law was still valid in at least some circumstances, and thus force strength is very important. Offensive, defensive, and staying power all contribute to the location of the parity point and thus are also valuable in determining success, whatever the military objectives;

(c) In high lethality combat, improved offensive power is key to avoiding outright defeat, while improvements to defensive and/or staying power contribute to victory (see the ratio locations on Figure 2); this is the reverse of the low lethality case. Force strength is important but problematic, as its contribution to mission success depends on the mission's objective and the tactics used; and,

(d) A navy that prefers the features of low lethality combat (for strategic, social, or other reasons) should increase the defensive power of its units, while one that prefers the nature of high lethality combat should boost the offensive power of its units. 
We should note that our concept of lethality herein is related to but distinct from several of the measures defined in [3]. One of those measures is combat work, which is the quantity of enemy units eliminated by a force during one salvo, e.g. the combat work done by $\mathrm{A}$ is $\Delta B=(\alpha A-B z) /(x)$. Like our lethality definition, combat work in a sense compares offensive power to both defensive and staying power, but there are important differences between these concepts. Combat work is calculated for a particular pair of force strengths $A \& B$, and due to these strengths could be high when lethality is low, or vice versa. By contrast, our lethality categories describe the character of combat over a range of possible force strengths. While the lethality level does depend on the characteristics of the two opposing unit types involved, it is independent of the quantities $A$ and $B$ committed to a particular battle. Thus lethality would be more useful say in the analysis of deployment options within an overall theatre of operations (where each side's force strengths for a possible engagement remain to be decided), while strength-specific measures such as combat work might be more relevant in the analysis of a particular battle within that theatre once forces have been committed.

\section{CONCLUSION}

In this article we proposed the idea of using the lethality level of a battle as a meaningful way to classify and understand combat between two homogenous forces. Our classification is based upon comparisons of the relative offensive and defensive characteristics of the unit types involved, and provides for three distinct levels of combat lethality. These definitions can be applied in the context of either a continuous time differential equation model or a discrete time salvo model. We compared the behavior of combat at each level of lethality, including the losses on each side and the relative difficulty of achieving some prototypical mission objectives. We argued that while moderate intensity combat behaves in a fairly straightforward manner (i.e., similar to what we have come to expect with traditional models of naval gun combat), low and high intensity combat each exhibits its own less intuitive behaviors. For example, the simple philosophy of "more is better" serves as a good rule of thumb when committing forces to moderate lethality combat, but requires re-thinking when a low or high lethality match-up of unit types arises. Incidental to our other results we also provided (in the appendix) the solution to a continuous time differential equation model that includes the defensive power feature of the discrete time salvo model.

There are a number of limitations to our work herein, of which probably the most important is that we assume (as in the continuous time [8] and discrete time models [3] that we build upon) that combat is deterministic without any element of chance. This is clearly unrealistic, as it can happen for example that the SAMs are "unlucky" enough to miss an incoming SSM and it allow it to "leak" through the defensive screen. This deterministic simplification is particularly misleading when the two sides are closely matched; [1] contains a good discussion of this issue. Thus one avenue for future research is the development of a stochastic version of this work; presumably this could build on existing research with stochastic differential equation models (e.g. [9]) and on a stochastic version of the salvo model (e.g. [6]). 
Another limitation is due to the aggregation of all units on each side into a single monolithic force; this effectively assumes that all incoming fire and resulting damage are spread evenly over all possible targets. In practice it is likely that some ships will face more fire than others, whether by accident or by design. For example, an attacker may deliberately concentrate fire on one vessel in order to penetrate a tough defensive screen in low lethality combat. Conversely, in high lethality combat a defender might deploy one vessel in advance of the main task force as a deliberate sacrifice to absorb a disproportionate number of incoming missiles. Thus future studies might explore the effective use of tactics and targeting information, much as was done in [7].

\section{ACKNOWLEDGEMENTS}

Thanks are due to two anonymous referees for their suggestions on improving the exposition of this work and for pointing out several additional references. During this research the author was funded in part by a Discovery Grant from the Natural Sciences and Engineering Research Council (NSERC) of Canada.

\section{APPENDIX}

\section{Derivation of Ratios}

The parity ratio $P$ is found by supposing that both sides eliminate the same proportion of the opponent's force $(\triangle B / B=\Delta A / A)$ and then solving for the ratio $B / A$.

$$
\begin{gathered}
(A \alpha-B z) / B x=(B \beta-A y) / A w \\
A w(A \alpha-B z)-B x(B \beta-A y)=0 \\
w(\alpha-z B / A)-x B / A(\beta B / A-y)=0 \\
x \beta(B / A)^{2}+(w z-x y)(B / A)-w \alpha=0
\end{gathered}
$$

Solve the quadratic equation and then continue re-arranging terms.

$$
\begin{gathered}
B / A=\left(-[w z-x y]+\left[(w z-x y)^{2}-4(x \beta)(-w \alpha)\right]^{1 / 2}\right) / 2 \beta x \\
=\frac{(\alpha w)^{1 / 2}-(w z-x y)+\left[(w z-x y)^{2}+4 \alpha \beta w x\right]^{1 / 2}}{(\beta x)^{1 / 2}} \\
=\sqrt{\frac{\alpha}{\beta} \frac{w}{x}\left(1+\frac{(w z-x y)^{2}-(w z-x y)\left[(w z-x y)^{2}+4 \alpha \beta w x\right]^{1 / 2}}{2 \alpha \beta w x}\right)} \\
=\sqrt{\frac{\alpha}{\beta} \frac{w}{x}(1+s)}, \quad \text { where } s \equiv \frac{(w z-x y)^{2}-(w z-x y)\left[(w z-x y)^{2}+4 \alpha \beta w x\right]^{1 / 2}}{2 \alpha \beta w x} .
\end{gathered}
$$

Ratios $R_{L}$ and $R_{U}$ for the favorable attrition boundaries are found by supposing that one force is eliminated and the losses to the other are in the same proportion as at $P$. Thus for the upper ratio $R_{U}$ we start with $\Delta B=-P A$ and solve for $B / A$. 


$$
\begin{gathered}
-\frac{A \alpha-B z}{x}=-P A \\
A \alpha-B z-P x A=0 \\
\alpha-z B / A-x P=0 \\
\frac{B}{A}=\frac{\alpha-x P}{z}
\end{gathered}
$$

The ratio $R_{L}$ is obtained in a similar manner by beginning with $\Delta A=-B / P$.

\section{Solutions of Differential Equations}

Given the initial strengths $A(0)$ and $B(0)$ of each side, the continuous time model becomes an initial value problem for a system of linear differential equations. The explicit solution has two state equations describing the remaining strength of each side.

$$
\begin{aligned}
& A(t)=\frac{(\beta x P+w z-x y) A(0)+\beta x B(0)}{2 \beta x P+w z-x y} e^{\frac{x y-\beta x P}{w x} t}-\frac{-\beta x P A(0)+\beta x B(0)}{2 \beta x P+w z-x y} e^{\frac{w z+\beta x P}{w x} t} \\
& B(t)=\frac{\beta x P B(0)+\alpha w A(0)}{2 \beta x P+w z-x y} e^{\frac{x y-\beta x P}{w x} t}-\frac{(-\beta x P-w z+x y) B(0)+\alpha w A(0)}{2 \beta x P+w z-x y} e^{\frac{w z+\beta x P}{w x} t}
\end{aligned}
$$

These equations apply until the earliest of the following events occurs:

(a) One side is eliminated, so either $A(t)=0$ or $B(t)=0$; or,

(b) One side is no longer able to hit the other due to an inability to penetrate its defenses, so that either $d / d t A(t)=0$ or $d / d t B(t)=0$.

Case (a) also arises in standard CLO models, and indicates that the battle is over. Case (b) on the other hand is due to the existence of defensive power; if for example $d / d t$ $B(t)=0$ occurs first at some time $t=t_{B}$, then the battle enters a second phase where A continues to suffer losses while $B$ remains stable at $B\left(t_{B}\right)$. A new differential equation describing A's losses would then take effect for the second phase of the battle.

$$
\frac{d}{d t} A(t)=-\left[\beta B\left(t_{B}\right)-y A(t)\right] / w, \quad \frac{d}{d t} A(t) \leq 0 ; \quad \frac{d}{d t} B(t)=0 ; \quad \text { for } t \geq t_{B}
$$

Solution yields the following description for remaining strength over time.

$$
A(t)=\frac{\beta}{y} B\left(t_{B}\right)-\left(\frac{\beta}{y} B\left(t_{B}\right)-A\left(t_{B}\right)\right) e^{\frac{y}{w} t}, \quad A(t) \geq 0 ; \quad B(t)=B\left(t_{b}\right) ; \quad \text { for } t \geq t_{B} .
$$




\section{REFERENCES}

[1] Ancker CJ, 1995, A proposed foundation for a theory of combat, Naval Research Logistics 42, 311-343.

[2] Armstrong MJ, 2001, One-on-one stochastic duels with suppression, Military Operations Research 6, \#4, 31-42.

[3] Hughes WP, 1995, A salvo model of warships in missile combat used to evaluate their staying power, Naval Research Logistics 42, 267-289.

[4] Hughes WP, 1995, Two effects of firepower: attrition and suppression, Military Operations Research 1, \#3, 27-35.

[5] Hughes WP, 2000, Fleet tactics and coastal combat, Naval Institute Press, Annapolis.

[6] Johns MD, Pilnick SE, Hughes WP, 2001, Heterogeneous salvo model for the navy after next, technical report NPS-IWJA-01-010, Naval Postgraduate School, Monterey.

[7] Lucas TW, McGunnigle JE, 2003, When is model complexity too much? Illustrating the benefits of simple models with Hughes' salvo equations, Naval Research Logistics 50, 197-217.

[8] Taylor JG, 1983, Lanchester Models of Warfare, Operations Research Society of America.

[9] Wiper MP, Pettit LI, Young KDS, 2000, Bayesian inference for a Lanchester type combat model, Naval Research Logistics 47, 541-558. 